\title{
High expression of the transcriptional coactivator TAZ is associated with a worse prognosis and affects cell proliferation in patients with medulloblastoma
}

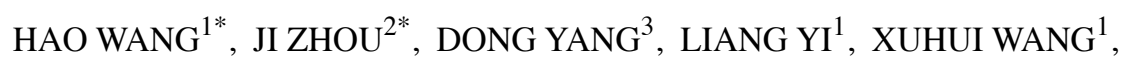 \\ YANGQING OU ${ }^{1}$, DONGHONG YANG ${ }^{1}$, LUNSHAN XU $^{1}$ and MINHUI XU ${ }^{1}$ \\ ${ }^{1}$ Department of Neurosurgery, Daping Hospital, Third Military Medical University, Chongqing 400042; ${ }^{2}$ Department of \\ Neurosurgery, Rocket Force General Hospital, Chinese People's Liberation Army, Beijing 100088; \\ ${ }^{3}$ Department of Healthy Management, Research Institute of Surgery, Daping Hospital, \\ Third Military Medical University, Chongqing 400042, P.R. China
}

Received April 14, 2018; Accepted April 11, 2019

DOI: $10.3892 / \mathrm{ol} .2019 .10851$

\begin{abstract}
The transcriptional coactivator tafazzin (TAZ) serves pivotal roles in organ development, tumor initiation and tumor progression. However, to the best of our knowledge, the expression of TAZ and its clinical significance in human medulloblastoma have not been defined. The present study aimed to clarify the clinical and biological significance of TAZ expression in human medulloblastoma. Immunohistochemical staining for TAZ was performed with 72 medulloblastoma and three normal brain tissue samples. A high expression level of TAZ was detected in $65.28 \%$ of medulloblastoma tissues, whereas low expression was identified in the normal brain tissues. TAZ expression was significantly associated with medulloblastoma recurrence. However, the expression of TAZ was not associated with sex, age, tumor location, tumor maximal diameter and tumor histology. Furthermore, both the overall survival and tumor-free survival rate of patients with high levels of expression of TAZ were shorter compared with those of patients with tumors expressing low levels of TAZ. In univariate and multivariate Cox regression analyses, TAZ expression was identified as a significant prognostic factor for patients with medulloblastoma. Functionally, downregulation of TAZ inhibited the proliferation and tumor formation of medulloblastoma cells and the expression of cell-cycle associated proteins in Daoy cells. In conclusion, high expression
\end{abstract}

Correspondence to: Dr Minhui Xu or Dr Lunshan Xu, Department of Neurosurgery, Daping Hospital, Third Military Medical University, 10 Changjiang Zhi Road, Daping, Yuzhong, Chongqing 400042, P.R. China

E-mail: xuminhui66@aliyun.com

E-mail: xulunshan@sina.com

*Contributed equally

Key words: tafazzin, medulloblastoma, prognosis, cell proliferation of TAZ may serve as a prognostic marker for patients with medulloblastoma and TAZ may be a potential target for medulloblastoma therapy.

\section{Introduction}

Medulloblastoma is the most common type of brain tumor in children and arises in the cerebellum (1). Current therapeutic approaches include surgical resection, craniospinal irradiation and chemotherapy, all of which are associated with severe long-term side effects, including intellectual damage and endocrine dysfunction (2-6). Identification of molecular markers that can be used to monitor the therapeutic outcomes of patients with medulloblastoma and decreasing the long-term sequelae with improved molecular-targeted therapies is required. Despite progress from recent studies regarding prognostic molecular markers for medulloblastoma, numerous patients still require an appropriate clinical prognostic marker (7-10).

The Hippo signaling pathway is a major regulator of tissue growth and organ size (11). The major function of this signaling pathway is largely determined by the downstream transcriptional regulator tafazzin (TAZ), which is a transcriptional co-activator with a PDZ-binding domain $(11,12)$. TAZ, also termed WW domain-containing transcription regulator 1 , serves pivotal roles in organ development $(13,14)$. TAZ functions by cooperating with several transcriptional factors, including T-box 5, paired box-8 and TEA domain family members (15-17). Given its biological effects in development and tissue homeostasis, TAZ has been a point of interest in cancer development and progression studies. The dysregulation of TAZ leads to concurrent uncontrolled cellular proliferation, inhibition of apoptosis and enhanced migration and invasion (18-20). In addition, the activity of TAZ is required to sustain the self-renewal and tumor-initiation capacities of cancer stem cells (CSCs). Cordenonsi et al (21) reported that the levels of TAZ protein are elevated in breast cancer CSCs and in poorly differentiated human malignant tumors. Additionally, a gain of TAZ was identified to induce a self-renewal capacity in non-CSCs. 
Despite this interest in elucidating the role of TAZ in cancer biology, to the best of our knowledge, the impact of TAZ expression on the prognosis and biological behavior of human medulloblastoma has not been investigated. The present study aimed to evaluate the expression of TAZ protein in medulloblastoma by immunohistochemistry and analyzed the association of TAZ expression with clinical outcomes by multivariate analysis. Furthermore, the functional role of TAZ in the proliferation of medulloblastoma cells was investigated.

\section{Materials and methods}

Patient tumor tissues. The use of tumor tissues from patients with medulloblastoma in the current study was approved by the Ethics Committee of Daping Hospital (Chongqing, China). A total of 72 patients with medulloblastoma, who initially underwent excision of their tumor without any other prior treatment, were enrolled between June 2005 and June 2012 at Daping Hospital or the Second Affiliated Hospital of Dalian Medical University (Dalian, China). These patients included 50 males and 22 females, (range, 5-43 years, mean 14.2 \pm 7.4 years). From these 72 patients with medulloblastoma, 55 patients were between the ages of 5 and 16 years, with a mean average age of $10.9 \pm 3.4$ years, and the remaining 17 patients ranged between 17 and 43 years, with an mean average age of 24.6 \pm 7.4 years. All patients were followed up until May 2014. The detailed characteristics of the patients are presented in Table I. The present study was performed according to the Declaration of Helsinki and written informed consent was obtained from all patients with tumors and donors of normal brain tissue samples.

Immunohistochemistry. For immunohistochemistry, preserved $10 \%$ formalin-fixed $\left(4^{\circ} \mathrm{C}, 6 \mathrm{~h}\right)$ and paraffin-embedded tissue samples, including human and mouse medulloblastoma tissue and normal tissue samples were cut into 5-mm-thick sections . Following deparaffinization in xylene, the sections were rehydrated in a graded alcohol series at room temperature (100, 95, 85 and 75\% ethanol). Endogenous peroxidase activity was blocked 15 min by incubation with $3 \%$ hydrogen peroxide in methanol at room temperature, after which the sections were microwaved $\left(100^{\circ} \mathrm{C}\right)$ in $0.01 \mathrm{M}$ sodium citrate buffer to retrieve the epitopes. The sections were incubated sequentially with primary antibodies against TAZ (dilution, 1:200; BD Biosciences; cat. no., 560235), and Ki-67 (dilution, 1:100; BD Biosciences; cat. no., 550609) overnight at $4^{\circ} \mathrm{C}$ and goat anti-mouse antibodies (dilution, 1:100; Santa Cruz Biotechnology, Inc; cat. no., sc-3738) for $30 \mathrm{~min}$ at room temperature. The sections were then incubated with 3,3'-diaminobenzidine for chromogen detection. A total of 10 randomly selected microscopy fields were observed under a light microscope (magnification, x20; Olympus-CKX41; Olympus Corporation, Tokyo, Japan) and the mean number of positive cells was calculated. The immunohistochemistry images were obtained under identical microscope conditions and magnification (x20).

Hematoxylin and eosin $(H \& E)$ staining. For immunohistochemistry, preserved $10 \%$ formalin-fixed $\left(4^{\circ} \mathrm{C}, 6 \mathrm{~h}\right)$ and paraffin-embedded tissue samples, including human and mouse medulloblastoma tissue and normal tissue samples were cut into 5-mm-thick sections, and stained with H\&E staining using the Hematoxylin and Eosin Staining Kit (Beyotime Institute of Biotechnology; cat. no., C0105), according to the manufacturer's protocols.

Semi-quantitative scoring of immunoreactivity. All tumor sections analyzed in the present study were reviewed by two pathologists (Chenyi Mao and Hualiang Xiao; Daping Hospital, Third Military Medical University). Tumor cell immunoreactivity was scored according to intensity and the extent of staining. The extent of immunopositivity was scored as follows: $1,<30 \%$ positive cells; $2,30-50 \%$ positive cells; and $3,>50 \%$ positive cells. For statistical analysis, sections with scores of 1 were considered to exhibit low expression and those with scores of 2 and 3 were considered to exhibit high expression.

Cell culture and lentivirus-mediated stable knockdown of TAZ expression. The medulloblastoma cell line Daoy was obtained from the American Type Culture Collection (Manassas, VA, USA) and cultured in Dulbecco's modified Eagle's medium/F12 (DMEM/F-12; Thermo Fisher Scientific, Inc.), supplemented with $10 \%$ fetal bovine serum (Thermo Fisher Scientific, Inc.), $1 \%$ penicillin and streptomycin. The GFP plasmid and TAZ-targeting short hairpin RNA (shRNA) in pLKO.1-Puro lentiviral vectors were kindly provided by Dr Heng Xiao (Department of Hepatobiliary and Pancreatic Surgery, First Affiliated Hospital of Zhejiang University, Zhejiang, China). Before transfection, 5x10 6 293FT cells (American Type Culture Collection) were plated on $100-\mathrm{mm}$ plate with lentiviral medium and incubated at $37^{\circ} \mathrm{C}$ overnight, Then $0.5 \mu \mathrm{g}$ pLKO.1 vector were mixed with $0.5 \mu \mathrm{g}$ of $\mathrm{pLP} 1, \mathrm{pLP} 2$ and $\mathrm{pLP} / \mathrm{VSVG}$ (packing) plasmids, and diluted in $6 \mu 1$ Lipofectamine $^{\mathrm{TM}} 2000$ (Thermo Fisher Scientific, Inc.) with $250 \mu$ l OPTI medium (no serum, penicillin and streptomycin; Thermo Fisher Scientific, Inc.). After $20 \mathrm{~min}$ at room temperature, the mixture was added dropwise into each 6 well plate, and $850 \mu$ l of the 293FT cell suspension ( $1 \times 10^{6}$ cells) was added to the plate containing OPTI medium and DNA-Lipofectamine ${ }^{\mathrm{TM}} 2000$ complexes. It was mixed gently by rocking the plate back and forth, and incubated at $37^{\circ} \mathrm{C}$ with $5 \% \mathrm{CO}_{2}$. The medium was replaced with lentiviral medium after 6-8 h transfection. After $48 \mathrm{~h}$, virus-containing supernatants were harvested, passed through a $0.45-\mu \mathrm{m}$ filter, and then infected with target cells. Cells stably expressing GFP and TAZ shRNA were generated after 3 days of culture in the presence of puromycin $(3 \mu \mathrm{g} / \mathrm{ml})$.

MTT assay. Cell viability was examined using the MTT assay. Cells $\left(1 \times 10^{3}\right.$ cells/well) were seeded in 96 -well plates with three replicates, and then were detected between day 0 and day 5. Purple formazan was dissolved by dimethylsulfoxide, and the absorbance was measured at a wavelength of $490 \mathrm{~nm}$. using a microplate reader. Each experiment was performed independently at least three times.

5-Bromo-2-deoxyuridine (BrdU) staining. For BrdU staining, $2 \times 10^{4}$ cells were seeded on coverslips in 24-well plates. Then cells were incubated with $10 \mu \mathrm{g} / \mathrm{ml}$ BrdU (Sigma-Aldrich; Merck $\mathrm{KGaA}$ ) for $30 \mathrm{~min}$ at $37^{\circ} \mathrm{C}$ with $5 \% \mathrm{CO}_{2}$ and fixed in 
Table I. Demographics of the 72 patients with medulloblastoma.

\begin{tabular}{|c|c|}
\hline Characteristic & $\mathrm{n}(\%)$ \\
\hline \multicolumn{2}{|l|}{ Sex } \\
\hline Male & $50(69.44)$ \\
\hline Female & $22(30.56)$ \\
\hline \multicolumn{2}{|l|}{ Age, years } \\
\hline$\leq 16$ & $55(76.39)$ \\
\hline$>16$ & $17(23.61)$ \\
\hline \multicolumn{2}{|l|}{ Symptoms } \\
\hline Headache & $57(79.17)$ \\
\hline Vomiting & $15(20.83)$ \\
\hline Ataxia & $5(6.94)$ \\
\hline Dizziness & $8(11.11)$ \\
\hline Leg weakness & $2(2.78)$ \\
\hline Visual symptoms & $2(2.78)$ \\
\hline \multicolumn{2}{|l|}{ Tumor maximal diameter, $\mathrm{cm}$} \\
\hline$\leq 2$ & $12(16.67)$ \\
\hline$>3, \leq 5$ & $53(73.61)$ \\
\hline$>5$ & $7(9.72)$ \\
\hline \multicolumn{2}{|l|}{ Tumor location } \\
\hline Hemisphere & $27(37.50)$ \\
\hline Vermis & $45(62.50)$ \\
\hline Metastases at diagnosis & $23(31.94)$ \\
\hline \multicolumn{2}{|l|}{ Extent of resection } \\
\hline Gross total & $46(63.89)$ \\
\hline Subtotal $^{\mathrm{a}}$ & $26(36.11)$ \\
\hline Recurrence & $53(73.61)$ \\
\hline Tumor bed & $19(26.39)$ \\
\hline Metastatic & $26(36.11)$ \\
\hline Mixed & $8(11.11)$ \\
\hline \multicolumn{2}{|l|}{ Postoperative treatment } \\
\hline Surgery only & $17(23.61)$ \\
\hline Surgery and irradiation & $34(47.22)$ \\
\hline Irradiation and chemotherapy & $21(29.17)$ \\
\hline \multicolumn{2}{|l|}{ Histology } \\
\hline Classical & $51(70.83)$ \\
\hline Nodular-desmoplastic & $9(12.50)$ \\
\hline Large cell/anaplastic & $12(16.67)$ \\
\hline Patient survival & $27(37.50)$ \\
\hline Patient mortality & $45(62.50)$ \\
\hline
\end{tabular}

Extent of resection, gross total resection as no residual tumour; ${ }^{a}$ Sub-total resection, $\geq 1.5 \mathrm{~cm}^{2}$ residual tumour; mixed, both in situ recurrence accompanied by distant metastasis.

4\% paraformaldehyde for $15 \mathrm{~min}$ at room temperature. The cells were then incubated with BrdU Detection Antibody kit (dilution, 1:300; Biovision, Inc.; cat. no., K306-1000) overnight at $4^{\circ} \mathrm{C}$, followed by the anti-mouse HRP-linked Antibody from the above BrdU Detection Antibody kit (dilution, 1:2,000; Biovision, Inc.) for $2 \mathrm{~h}$, according to the manufacturer's protocols. A total of $300 \mathrm{nM}$ DAPI was then added for counterstaining, according to the BrdU Detection Antibody kit. The images were captured using a light microscope (magnification, x20) with Image-Pro Plus software (X-Ray, Celina; https://www.xrayscan.com/software-image-pro-plus/) for image analysis and green staining indicated BrdU-positive cells.

Western blot analysis. Cellular proteins were extracted with RIPA lysis buffer (Thermo Fisher Scientific, Inc.). The protein concentration of the proteins was determined by the BCA Protein Assay kit (Beijing Solarbio Science \& Technology Co., Ltd.; cat. no., PC0020). Equal amounts of protein $(50 \mu \mathrm{g})$ were subjected to $10 \%$ SDS-PAGE and electrotransferred onto a hydrophobic PVDF membrane (Roche Diagnostics). Membranes were subsequently blocked with $5 \%$ nonfat milk in TBST $(0.1 \%$ Tween $)$ for $2 \mathrm{~h}$ at room temperature and subsequently incubated with primary antibodies against TAZ (dilution, 1:2,000; BD Biosciences; cat. no., 560235), tubulin (dilution, 1:3,000; Beyotime Institute of Biotechnology; cat.no., A01080HRP), cyclin-dependent kinase 4 (CDK4, dilution, 1:200; Santa Cruz Biotechnology, Inc.; cat. no., sc-70831), cyclin D1 (CCND1, dilution, 1:200; Santa Cruz Biotechnology, Inc.; cat. no., sc-8396) and cyclin E2 (CCNE2, dilution, 1:200; Santa Cruz Biotechnology, Inc.; cat. no., sc-28351) under slow rotation overnight at $4^{\circ} \mathrm{C}$. Subsequently, the membranes were incubated with the corresponding secondary antibodies HRP-labeled goat anti-mouse IgG (H+L) (cat. no., A0216; dilution, 1:5,000; Beyotime Institute of Biotechnology) and goat anti-rabbit $\operatorname{IgG}(\mathrm{H}+\mathrm{L})$ (cat. no., A0216; dilution, 1:5,000; Beyotime Institute of Biotechnology) for $2 \mathrm{~h}$ at $4^{\circ} \mathrm{C}$. They were subsequently captured using the ECL Reagent (Cell Signaling Technology, Inc.) and visualized by iBright Imaging Systems (iBright FL 1000; Thermo Fisher Scientific, Inc.).

Xenograft assay. A total of 12 female SCID mice (4 weeks old; 18-20 g) were purchased from Beijing Laboratory Animal Research Center and housed in an SPF room, which maintained standard housing conditions with $12 \mathrm{~h}$ light-dark cycle with food and water available ad libitum and at a constant temperature $\left(22^{\circ} \mathrm{C}-25^{\circ} \mathrm{C}\right)$ and humidity $(40-50 \%)$. The human medulloblastoma Daoy cells $\left(1 \times 10^{6}\right)$ stably transfected with sh-GFP or sh-TAZ were injected slowly into the subcutis on the dorsum of each mouse. The tumor size was monitored every week using a vernier caliper and tumor volume (V) was calculated using the formula: $\mathrm{V}=\left(\right.$ length $\mathrm{x}$ width $\left.{ }^{2}\right) / 2$. At the termination of the experiment (day 45), the tumor mass was harvested, weighed and stored for immunohistochemical analysis. All studies were approved by the Ethics Committee of Daping Hospital (Chongqing, China).

Statistical analysis. All statistical analyses were performed using SPSS 11.5 software (SPSS, Inc., Chicago, IL, USA). Differences in the levels of TAZ expression among various subgroups were analyzed using a $\chi^{2}$ test. The overall survival and tumor-free survival rates were evaluated using Kaplan-Meier analysis and the survival of groups of patients was compared using a log-rank test. All experiments were performed in triplicate and the quantitative data are presented 

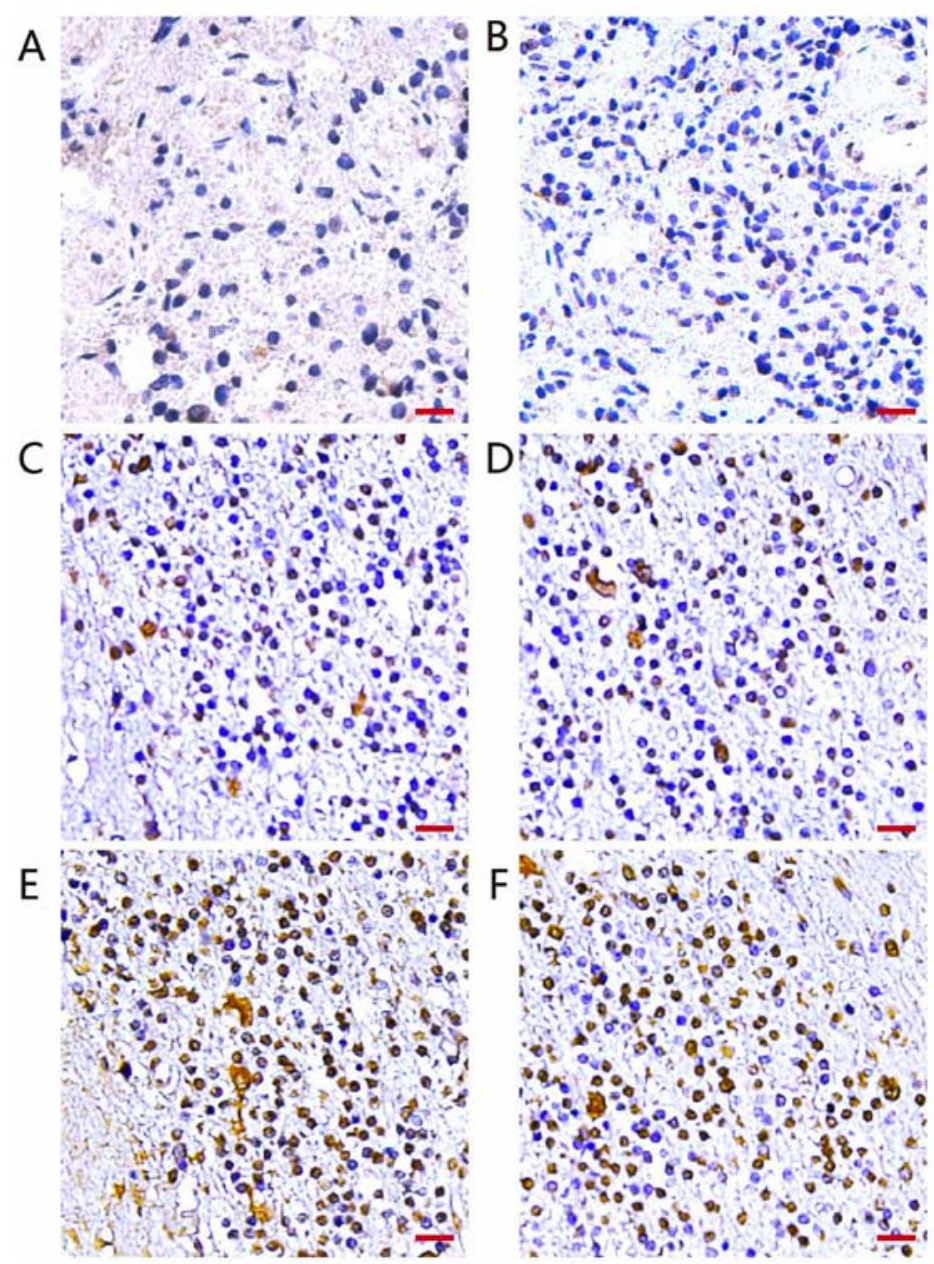

Figure 1. Detection of TAZ by immunohistochemical staining of medulloblastoma and normal brain tissue samples. (A) Weak TAZ expression in normal brain tissue. (B) Weak TAZ expression in medulloblastoma tissue. (C and D) Representative figures of moderate TAZ expression in 25 medulloblastoma tissues. (E and F) Representative figures of strong TAZ expression in 47 medulloblastoma tissue. Scale bars $=100 \mu \mathrm{m}$. TAZ, tafazzin.

as the mean \pm standard deviation. Two-tailed Student's t-tests were performed to calculate significance with a $95 \%$ confidence level in data with a normal distribution and different but similar standard deviations. $\mathrm{P}<0.05$ was considered to indicate a statistically significant difference.

\section{Results}

Immunostaining of TAZ expression in medulloblastoma tissues. Immunohistochemistry was performed to detect TAZ expression in 72 medulloblastoma and three normal brain tissue samples. The results revealed low expression of TAZ in the three normal brain tissues (Fig. 1A). By contrast, TAZ expression, which was predominantly observed in the nuclei, was elevated in a high proportion of medulloblastoma tissues. In total, 25 of the tumors $(34.72 \%)$ demonstrated low expression of TAZ (Fig. 1B-D) and 47 of the tumors (65.28\%) demonstrated high expression (Fig. 1E and F; Table II). These results suggest TAZ may serve an important role in the progression of medulloblastoma.

Association of TAZ expression and clinicopathological features of patients with medulloblastoma. The TAZ immunohistochemistry results were analyzed for potential associations with clinicopathological characteristics of patients with medulloblastoma. As presented in Table II, no significant associations were identified between TAZ expression and sex $(\mathrm{P}=0.465)$, age $(\mathrm{P}=0.522)$, tumor location $(\mathrm{P}=0.848)$, tumor maximal diameter $(\mathrm{P}=0.912)$, histological type $(\mathrm{P}=0.212)$ or tumor metastases at diagnosis $(\mathrm{P}=0.292)$. However, the expression of TAZ was significantly associated with tumor recurrence. Among the 52 patients who had recurrence during follow-up, 38 patients exhibited a high expression of TAZ and the others had low expression. The recurrence rate in patients with tumors with high TAZ expression was $80.9 \%$, whereas the rate in patients with tumors with low TAZ-expressing tumors was only $56 \%(\mathrm{P}=0.025)$.

Association of TAZ expression with overall survival and tumor-free survival. As presented in Fig. 2, the association of TAZ expression with overall survival and tumor-free survival rate was evaluated by log-rank test. Patients with high TAZ expression demonstrated significantly shorter overall survival and tumor-free survival times compared with patients with low TAZ expression (Fig. 2). Univariate and multivariate Cox regression analyses were performed to determine the independent prognostic factors for overall survival of patients with medulloblastoma. Univariate Cox regression 
Table II. Association of TAZ expression with clinicopathological features of patients with medulloblastoma.

\begin{tabular}{|c|c|c|c|}
\hline Characteristic & TAZ low expression $(n=25)$ & TAZ high expression $(n=47)$ & P-value \\
\hline $\operatorname{Sex}$ & & & 0.465 \\
\hline Male & 16 & 34 & \\
\hline Female & 9 & 13 & \\
\hline Age, years & 25 & 47 & 0.522 \\
\hline$\leq 16$ & 18 & 37 & \\
\hline$>16$ & 7 & 10 & \\
\hline Tumor maximal diameter, $\mathrm{cm}$ & & & 0.912 \\
\hline$\leq 3$ & 4 & 8 & \\
\hline$>3$ & 21 & 39 & \\
\hline Tumor location & & & 0.848 \\
\hline Hemisphere & 9 & 18 & \\
\hline Vermis & 16 & 29 & \\
\hline Metastases at diagnosis & 6 & 17 & 0.292 \\
\hline Recurrence & 14 & 38 & $0.025^{\mathrm{a}}$ \\
\hline Histology & & & 0.212 \\
\hline Classic & 20 & 31 & \\
\hline Non-classic & 5 & 16 & \\
\hline
\end{tabular}

${ }^{\text {aP }}<0.05$. TAZ, tafazzin.

Table III. Univariate Cox regression analysis of overall survival.

\begin{tabular}{|c|c|c|c|c|}
\hline Factor & Wald-value & $\operatorname{Exp}(B)$ & $95 \%$ CI for $\operatorname{Exp}(B)$ & $\mathrm{P}$-value \\
\hline TAZ expression & 3.952 & 2.042 & $1.010-4.130$ & $0.047^{\mathrm{a}}$ \\
\hline Sex & 0.002 & 1.015 & $0.509-2.023$ & 0.967 \\
\hline Age & 1.236 & 0.638 & $0.289-1.408$ & 0.266 \\
\hline Tumor location & 0.023 & 0.945 & $0.458-1.950$ & 0.879 \\
\hline Metastasis at diagnosis & 23.805 & 6.569 & $3.084-13.992$ & $<0.001^{\mathrm{a}}$ \\
\hline Tumor diameter & 0.238 & 1.263 & $0.494-3.232$ & 0.626 \\
\hline Resection extent & 2.862 & 1.714 & $0.918-3.198$ & 0.091 \\
\hline Tumor histology & 1.050 & 0.795 & $0.514-1.232$ & 0.305 \\
\hline Postoperative therapy & 6.426 & 0.557 & $0.355-0.876$ & $0.011^{\mathrm{a}}$ \\
\hline
\end{tabular}

${ }^{\mathrm{a}} \mathrm{P}<0.05$. CI, confidence interval; TAZ, tafazzin.
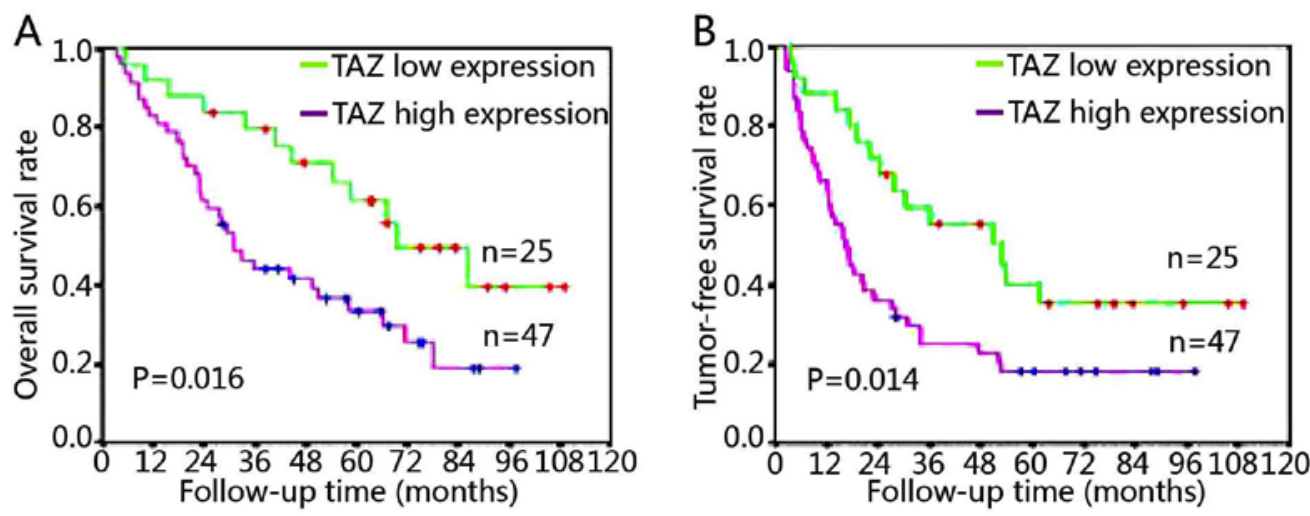

Figure 2. Survival analysis of patients with medulloblastoma according to the TAZ expression level. Kaplan-Meier survival analysis of (A) overall survival and (B) tumor-free survival rate of patients with a high or low tumor TAZ expression level. P-values were determined by a log-rank test. TAZ, tafazzin. 
Table IV. Multivariate Cox regression analysis of overall survival.

\begin{tabular}{lrccr}
\hline Factors & Wald-value & Exp(B) & $95 \%$ CI for Exp(B) & P-value \\
\hline TAZ expression & 3.965 & 2.008 & $1.011-3.986$ & $0.046^{\mathrm{a}}$ \\
Metastasis at diagnosis & 25.008 & 6.132 & $3.012-12.482$ & $<0.001^{\mathrm{a}}$ \\
Postoperative therapy & 5.987 & 0.587 & $0.383-0.899$ & $0.014^{\mathrm{a}}$ \\
\hline
\end{tabular}

${ }^{\mathrm{a}} \mathrm{P}<0.05$. CI, confidence interval; TAZ, tafazzin.

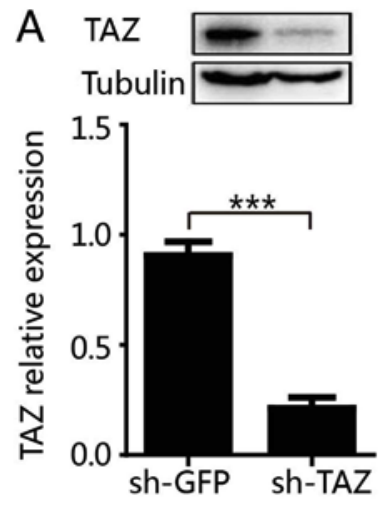

B
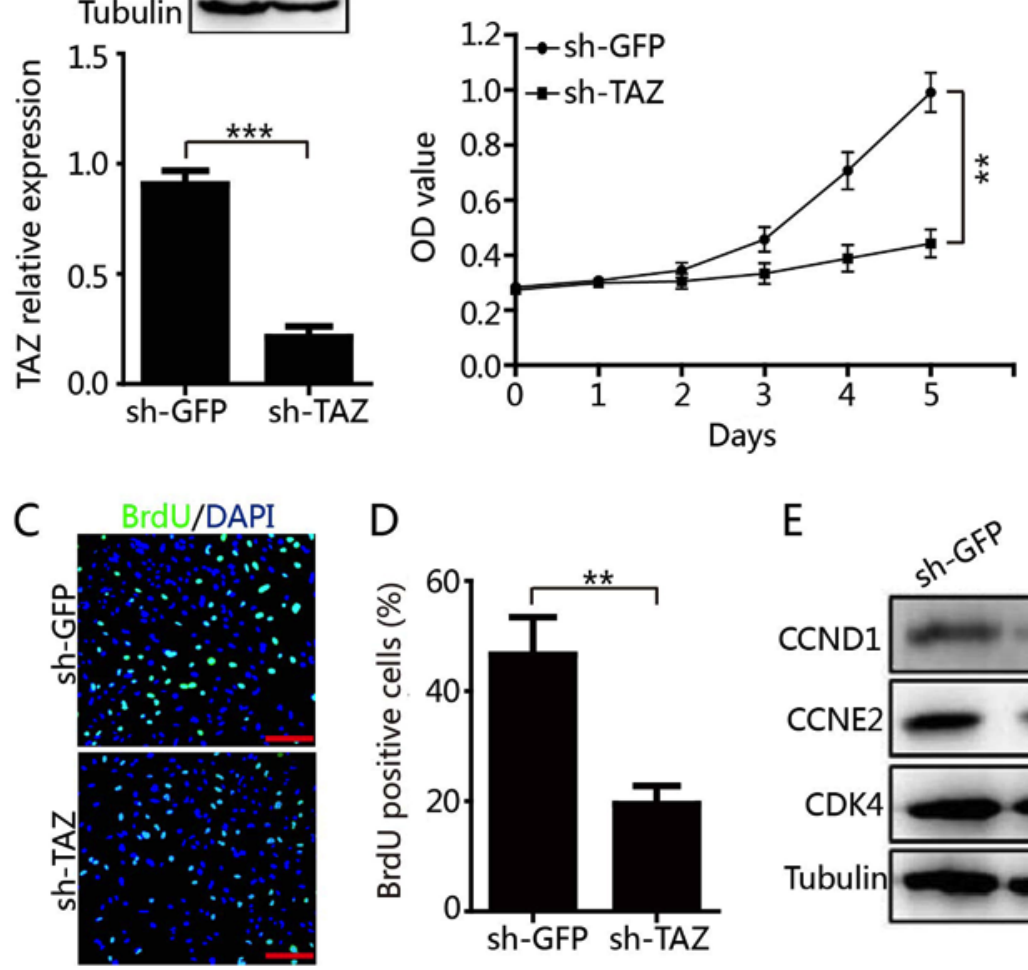

Figure 3. Downregulation of TAZ inhibits medulloblastoma Daoy cell proliferation. (A) The expression level of TAZ in TAZ-knockdown Daoy cells and control cells was analyzed by western blot analysis. (B) The effect of TAZ downregulation on medulloblastoma Daoy cell proliferation was examined by MTT assay. (C) A BrdU assay was performed to detect the proliferation ability following TAZ downregulation. Scale bar=100 $\mu \mathrm{m}$. (D) Quantification of the BrdU assay results. (E) The expression levels of cell-cycle-associated proteins were examined by western blot analysis. All data are presented as the mean \pm standard deviation. ${ }^{* *} \mathrm{P}<0.01,{ }^{* * *} \mathrm{P}<0.001$. TAZ, tafazzin; sh, short hairpin; CCND1, cyclin D1; CCNE2, cyclin E2; CDK4, cyclin-dependent kinase 4; OD, optical density.

analysis first revealed TAZ expression $(\mathrm{P}=0.047)$, metastasis at diagnosis $(\mathrm{P}<0.001)$ and postoperative therapy $(\mathrm{P}=0.011)$ as significant prognostic factors for patients with medulloblastoma (Table III). However, histological type and resection extent were not significant prognostic factors. Furthermore, multivariate analysis identified TAZ expression $(\mathrm{P}=0.046)$, metastasis at diagnosis $(\mathrm{P}<0.001)$ and postoperative therapy strategies $(\mathrm{P}=0.014)$ as independent prognostic factors for the overall survival of patients with medulloblastoma (Table IV).

Downregulation of TAZ inhibits cell proliferation of Daoy cells. Enhanced expression of TAZ was identified to be associated with short overall survival and tumor-free survival times of patients with medulloblastoma. However, the functional role of TAZ in medulloblastoma progression remains unknown. To address this issue, the present study established Daoy cells with stable expression of shRNA targeting TAZ (sh-TAZ) and control shRNA targeting GFP (sh-GFP). As presented in Fig. 3A, the protein level of TAZ was significantly downregulated in sh-TAZ cells compared with sh-GFP cells. Subsequently, the role of TAZ in the regulation of the proliferation of Daoy cells was examined. MTT assay demonstrated that knockdown of TAZ expression significantly inhibited the proliferation of Daoy cells (Fig. 3B). This result was further confirmed using a BrdU incorporation assay. The percentage of BrdU-positive cells was significantly decreased in sh-TAZ cells compared with sh-GFP cells (Fig. 3C and D). Finally, it was identified that the expression levels of CCND1 and CCNE2 were decreased in the sh-TAZ group compared with the sh-GFP group, while no obvious changes were observed in CDK4 expression (Fig. 3E). In summary, these results suggested that TAZ may 


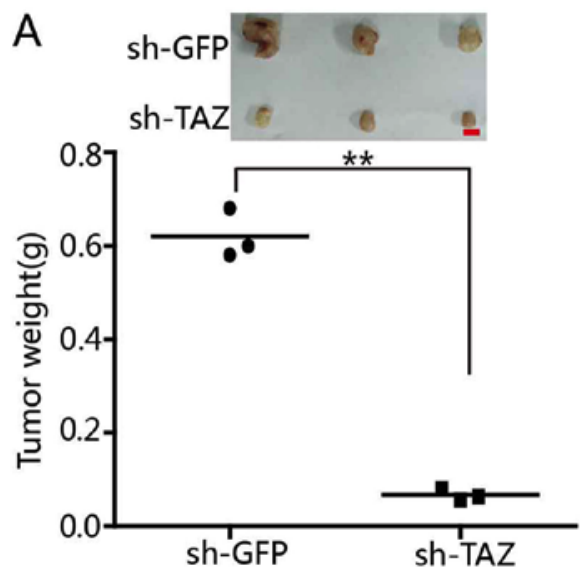

B
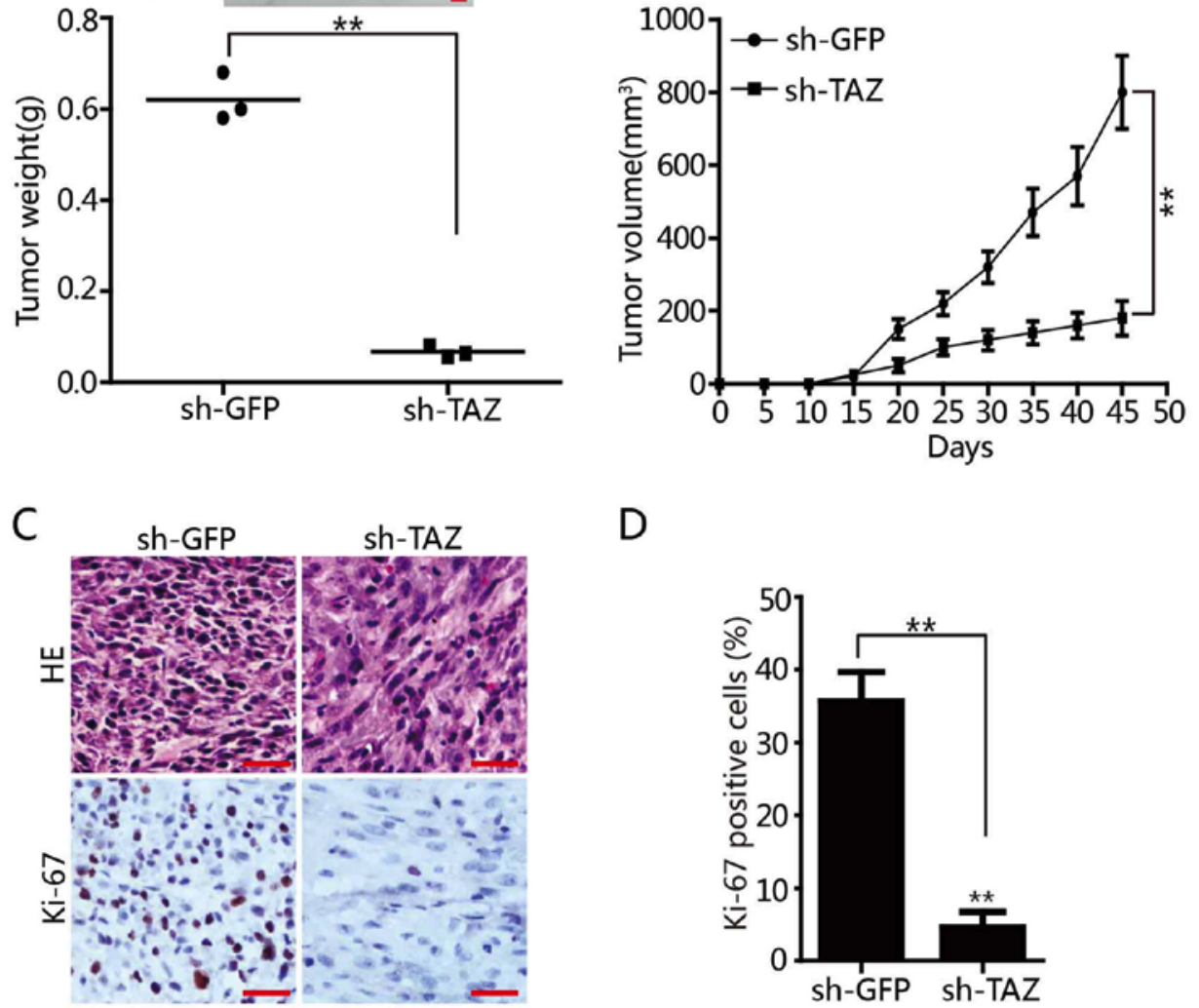

D

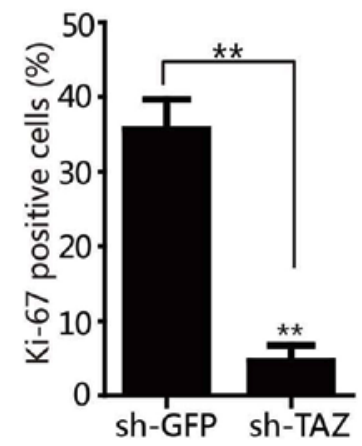

Figure 4. Downregulation of TAZ inhibits the tumor formation of Daoy cells in vivo. (A) The size and weight of xenograft tumor formed by the TAZ-knockdown Daoy cells and control cells. Scale bar=1 mm. (B) The tumor growth curve of TAZ-knockdown Daoy cells and control cells injected into immunodeficient mice. (C) Immunohistochemical staining for Ki-67 in tumor tissues. Scale bar=100 $\mu \mathrm{m}$. (D) Quantification of immunohistochemical staining. All data are presented as the mean \pm standard deviation. ${ }^{* *} \mathrm{P}<0.01$. sh, short hairpin; TAZ, tafazzin; H\&E, haemotoxylin and eosin.

regulate the proliferation and expression of cell-cycle-associated proteins in medulloblastoma cells, which indicates a role for TAZ in the progression of medulloblastoma.

Downregulation of TAZ inhibits the tumor formation of Daoy cells in vivo. To investigate the effects of TAZ expression on medulloblastoma tumor formation, a nude mouse xenograft experiment was performed. In the nude mouse xenograft experiment, tumor growth was significantly inhibited in nude mice injected with TAZ-knockdown Daoy cells compared with control mice (Fig. 4A and B). Immunohistochemical staining revealed that the expression of Ki-67, a cell proliferation marker, was significantly reduced in the tissues samples of mice injected with sh-TAZ cells compared with those injected with sh-GFP cells (Fig. 4C and D).

\section{Discussion}

Medulloblastoma, which arises in the cerebellum, is the most common type of malignant brain tumor in children (22). Despite progress in the treatment of medulloblastoma, $30 \%$ of patients will succumb to the disease and $40 \%$ of children diagnosed with the disease will experience tumor recurrence (23). Although conventional therapies can treat a large proportion of patients with medulloblastoma, the majority of survivors experience a reduced quality of life.

Current risk stratification strategies are largely based on clinical parameters, including age at diagnosis, metastatic status, extent of surgical resection and histological features (24). Molecular stratification and the use of molecular targeted therapy for patients with medulloblastoma is still in its infancy and has yet to be routinely implemented in the clinical practice (25). An improved understanding of the molecular and genetic basis of medulloblastoma will contribute to appropriate stratification and the treatment of patients based on biological markers, leading to improved patient outcomes with reduced sequelae.

Medulloblastoma is a heterogeneous tumor type consisting of at least four distinct subgroups, including the WNT subgroup, sonic hedgehog subgroup, subgroup $\mathrm{C}$ and subgroup D (26). This classification is based on immunohistochemistry data and has been widely used in medulloblastoma research, directing numerous studies toward elucidating the mechanisms of activation of WNT and SHH signaling pathways and the development of new strategies to inhibit them, thus leading to the improvement of patient stratification and prognosis (27-29). Additionally, the outcomes of patients with medulloblastoma have been reported to be associated with c-MYC copy number and the 
expression of microRNA-183/96/182 and cyclin-dependent kinase $6(30,31)$. However, additional markers that can be utilized for monitoring medulloblastoma progression and therapeutic outcome are required.

The present study examined the expression of TAZ in 72 patients with medulloblastoma. High TAZ expression was observed in $65.28 \%$ of patients. Low or undetectable immunostaining for TAZ was observed in normal brain tissues. Subsequently, it was demonstrated that expression of TAZ is a negative prognostic factor for the overall survival rate of patients with medulloblastoma. To the best of our knowledge, this is a new finding, as no previous studies have assessed the impact of TAZ expression on the prognosis of patients with medulloblastoma. The present results are consistent with studies of adenocarcinoma of the esophagogastric junction and colorectal cancer $(32,33)$. However, in the current study, a lower rate of patients with long-time survival was observed compared with a previous study (23). This may be due to the fact that fewer patients in the present study had received combined therapy (23.61 and $47.22 \%$ received surgery alone and combined surgery and irradiation, respectively). In addition, it was identified that TAZ expression was significantly associated with medulloblastoma recurrence. However, the current results are limited by the amount of clinical data and different patient follow-up periods. Therefore the sample size should be increased and the median follow-up time should be analyzed in future studies.

Additional functional experiments in the present study revealed that the expression of TAZ promoted tumor proliferation. Additionally, silencing TAZ expression decreased the proliferation and tumor formation of medulloblastoma cells. The present study highlights the important role of TAZ in medulloblastoma progression and its association with patient survival.

In summary, high expression of TAZ was detected in a majority of medulloblastoma tissue samples and was identified to predict a poor outcome for patients. As a result, the expression of TAZ may serve as a prognostic marker and a potential therapeutic target for patients with medulloblastoma. However, this conclusion is limited by the small number of cases analyzed in the present study. Further studies with a large number of cases are required to validate the clinical significance of TAZ in patients with medulloblastoma and elucidate the precise mechanism by which TAZ regulates the malignant behavior of medulloblastoma.

\section{Acknowledgements}

The authors would like to thank Dr Heng Xiao (Zhejiang University School of Medicine, Hangzhou, China) for technical support.

\section{Funding}

The present study was supported by the Natural Science Foundation of China (grant no. 81300965). The Natural Science Foundation of Chongqing (grant no. cstc2017jcyjAX0337) and the Chongqing Basic Science Frontier Technology Special Key Project (grant nos. cstc2017jcyjB0164, cstc2017jcyjBX0021 and cstc2018jcyjAX0600).

\section{Availability of data and materials}

The datasets used and/or analyzed during the present study are available from the corresponding author on reasonable request.

\section{Authors' contributions}

HW and JZ collected, analyzed and interpreted the data, and wrote the manuscript. DY, LY, XW, YO and DHY collected and analyzed the data. LX and MX designed the study, provided financial support, analyzed and interpreted the data, and revised the manuscript. All authors read and approved the final manuscript.

\section{Ethics approval and consent to participate}

All animal studies were approved by the Ethics Committee of Daping Hospital (Chongqing, China). The use of tissue samples from patients was approved by the Ethics Committee of Daping Hospital. The current study was performed according to the Declaration of Helsinki and written informed consent was obtained from all patients.

\section{Patient consent for publication}

Not applicable.

\section{Competing interests}

The authors declare that they have no competing interests.

\section{References}

1. Northcott PA, Robinson GW, Kratz CP, Mabbott DJ, Pomeroy SL, Clifford SC, Rutkowski S, Ellison DW, Malkin D, Taylor MD, et al: Medulloblastoma. Nat Rev Dis Primers 5: 11, 2019.

2. Ris MD, Walsh K, Wallace D, Armstrong FD, Holmes E, Gajjar A, Zhou T and Packer RJ: Intellectual and academic outcome following two chemotherapy regimens and radiotherapy for average-risk medulloblastoma: COG A9961. Pediatr Blood Cancer 60: 1350-1357, 2013.

3. Moxon-Emre I, Bouffet E, Taylor MD, Laperriere N, Scantlebury N, Law N, Spiegler BJ, Malkin D, Janzen L and Mabbott D: Impact of craniospinal dose, boost volume, and neurologic complications on intellectual outcome in patients with medulloblastoma. J Clin Oncol 32: 1760-1768, 2014.

4. Polkinghorn WR and Tarbell NJ: Medulloblastoma: Tumorigenesis, current clinical paradigm, and efforts to improve risk stratification. Nat Clin Pract Oncol 4: 295-304, 2007.

5. Saha A, Salley CG, Saigal P, Rolnitzky L, Goldberg J, Scott S, Olshefski R, Hukin J, Sands SA, Finlay J and Gardner SL: Late effects in survivors of childhood CNS tumors treated on Head Start I and II protocols. Pediatr Blood Cancer 61: 1644-1652; quiz 1653-1672, 2014.

6. Wang J, Garancher A, Ramaswamy V and Wechsler-Reya RJ: Medulloblastoma: From molecular subgroups to molecular targeted therapies. Annu Rev Neurosci 41: 207-232, 2018.

7. Liu KW, Pajtler KW, Worst BC, Pfister SM and Wechsler-Reya RJ: Molecular mechanisms and therapeutic targets in pediatric brain tumors. Sci Signal 10, 2017.

8. Robinson GW, Witt $\mathrm{H}$ and Resnick A: Exploiting laboratory insights to improve outcomes of pediatric central nervous system tumors. Am Soc Clin Oncol Educ Book 35: e540-e546, 2016.

9. Tamayo P, Cho YJ, Tsherniak A, Greulich H, Ambrogio L, Schouten-van Meeteren N, Zhou T, Buxton A, Kool M, Meyerson M, et al: Predicting relapse in patients with medulloblastoma by integrating evidence from clinical and genomic features. J Clin Oncol 29: 1415-1423, 2011. 
10. von Bueren AO, Kortmann RD, von Hoff K, Friedrich C, Mynarek M, Müller K, Goschzik T, Zur Mühlen A, Gerber N, Warmuth-Metz M, et al: Treatment of children and adolescents with metastatic medulloblastoma and prognostic relevance of clinical and biologic parameters. J Clin Oncol 34: 4151-4160, 2016.

11. Yu FX, Zhao B and Guan KL: Hippo pathway in organ size control, tissue homeostasis, and cancer. Cell 163: 811-828, 2015.

12. Moroishi T, Park HW, Qin B, Chen Q, Meng Z, Plouffe SW, Taniguchi K, Yu FX, Karin M, Pan D and Guan KL: A YAP/TAZ-induced feedback mechanism regulates Hippo pathway homeostasis. Genes Dev 29: 1271-1284, 2015.

13. Totaro A, Panciera T and Piccolo S: YAP/TAZ upstream signals and downstream responses. Nat Cell Biol 20: 888-899, 2018.

14. Maugeri-Saccà M and De Maria R: The Hippo pathway in normal development and cancer. Pharmacol Ther 186: 60-72, 2018.

15. Murakami M, Nakagawa M, Olson EN and Nakagawa O: A WW domain protein TAZ is a critical coactivator for TBX5, a transcription factor implicated in Holt-Oram syndrome. Proc Natl Acad Sci USA 102: 18034-18039, 2005.

16. Di Palma T, D'Andrea B, Liguori GL, Liguoro A, de Cristofaro T, Del Prete D, Pappalardo A, Mascia A and Zannini M: TAZ is a coactivator for Pax8 and TTF-1, two transcription factors involved in thyroid differentiation. Exp Cell Res 315: 162-175, 2009.

17. Zhang H, Liu CY, Zha ZY, Zhao B, Yao J, Zhao S, Xiong Y, Lei QY and Guan KL: TEAD transcription factors mediate the function of TAZ in cell growth and epithelial-mesenchymal transition. J Biol Chem 284: 13355-13362, 2009.

18. Zhang X, Zhao H, Li Y, Xia D, Yang L, Ma Y and Li H: The role of YAP/TAZ activity in cancer metabolic reprogramming. Mol Cancer 17: 134, 2018.

19. Liu H, Du S, Lei T, Wang H, He X, Tong R and Wang Y: Multifaceted regulation and functions of YAP/TAZ in tumors (Review). Oncol Rep 40: 16-28, 2018

20. Choi W, Kim J, Park J, Lee DH, Hwang D, Kim JH, Ashktorab H, Smoot D, Kim SY, Choi C, et al: YAP/TAZ initiates gastric tumorigenesis via upregulation of MYC. Cancer Res 78: 3306-3320, 2018.

21. Cordenonsi M, Zanconato F, Azzolin L, Forcato M, Rosato A, Frasson C, Inui M, Montagner M, Parenti AR, Poletti A, et al: The Hippo transducer TAZ confers cancer stem cell-related traits on breast cancer cells. Cell 147: 759-772, 2011.

22. Cassia GSE, Alves C, Taranath A, López NS, Oztekin O, Gonçalves FG and Patay Z: Childhood medulloblastoma revisited. Top Magn Reson Imaging 27: 479-502, 2018.

23. Rutkowski S, von Hoff K, Emser A, Zwiener I, Pietsch T, Figarella-Branger D, Giangaspero F, Ellison DW, Garre ML, Biassoni $\mathrm{V}$, et al: Survival and prognostic factors of early childhood medulloblastoma: An international meta-analysis. J Clin Oncol 28: 4961-4968, 2010.
24. Ramaswamy V, Remke M, Bouffet E, Bailey S, Clifford SC, Doz F, Kool M, Dufour C, Vassal G, Milde T, et al: Risk stratification of childhood medulloblastoma in the molecular era: The current consensus. Acta Neuropathol 131: 821-831, 2016.

25. Low JA and de Sauvage FJ: Clinical experience with Hedgehog pathway inhibitors. J Clin Oncol 28: 5321-5326, 2010.

26. Northcott PA, Korshunov A, Witt H, Hielscher T, Eberhart CG, Mack S, Bouffet E, Clifford SC, Hawkins CE, French P, et al: Medulloblastoma comprises four distinct molecular variants. J Clin Oncol 29: 1408-1414, 2011.

27. Kool M, Korshunov A, Remke M, Jones DT, Schlanstein M, Northcott PA, Cho YJ, Koster J, Schouten-van Meeteren A, van Vuurden D, et al: Molecular subgroups of medulloblastoma: An international meta-analysis of transcriptome, genetic aberrations, and clinical data of WNT, SHH, Group 3, and Group 4 medulloblastomas. Acta Neuropathol 123: 473-484, 2012.

28. ŁastowskaM,TrubickaJ,Niemira M,Paczkowska-Abdulsalam M, Karkucińska-Więckowska A, Kaleta M, Drogosiewicz M, Tarasińska M, Perek-Polnik M, Krętowski A, et al: ALK expression is a novel marker for the WNT-activated type of pediatric medulloblastoma and an indicator of good prognosis for patients. Am J Surg Pathol 41: 781-787, 2017.

29. Takei H, Bhattacharjee MB, Rivera A, Dancer Y and Powell SZ: New immunohistochemical markers in the evaluation of central nervous system tumors: A review of 7 selected adult and pediatric brain tumors. Arch Pathol Lab Med 131: 234-241, 2007.

30. Cho YJ, Tsherniak A, Tamayo P, Santagata S, Ligon A, Greulich H, Berhoukim R, Amani V, Goumnerova L, Eberhart CG, et al: Integrative genomic analysis of medulloblastoma identifies a molecular subgroup that drives poor clinical outcome. J Clin Oncol 29: 1424-1430, 2011.

31. Mendrzyk F, Radlwimmer B, Joos S, Kokocinski F, Benner A, Stange DE, Neben K, Fiegler H, Carter NP, Reifenberger G, et al: Genomic and protein expression profiling identifies CDK6 as novel independent prognostic marker in medulloblastoma. J Clin Oncol 23: 8853-8862, 2005.

32. Sun L, Chen F, Shi W, Qi L, Zhao Z and Zhang J: Prognostic impact of TAZ and $\beta$-catenin expression in adenocarcinoma of the esophagogastric junction. Diagn Pathol 9: 125, 2014.

33. Wang Z, Liu P, Zhou X, Wang T, Feng X, Sun YP, Xiong Y, Yuan HX and Guan KL: Endothelin promotes colorectal tumorigenesis by activating YAP/TAZ. Cancer Res 77: 2413-2423, 2017. 